\title{
TECNICAS ACTUALES PARA EL PROYECTO Y LA CONSTRUCCION DE LAS GRANDES CHIMENEAS INDUSTRIALES
}

\author{
(PRESENT TECHNIQUES TO PROJECT AND BUILT TALL INDUSTRIAL CHIMNEYS)
}

José del Solar Bermejo, Ingeniero de Caminos

Director técnico de KARMAN, Técnicas Especiales, S. A.

\section{RESUMEN}

Este trabajo trata de dar a conocer el profundo cambio experimentado en el diseño de las chimeneas industriales de gran altura.

Se describen las caracteristicas principales de este tipo de obras, y el proyecto con los medios informáticos empleados en los cálculos estructurales, termodinámicos y de dispersión de humos, para el dimensionamiento optimo de una chimenea industrial.

\section{SUMMARY}

This work intends to make known the deep change met within the design of great-height industrial chimneys.

The main characteristics of this kind of work are described, as well as the project with the informatic means used in structural,

thermodynamic and smoke dispersion calculations for the optimal design of an industrial chimney.

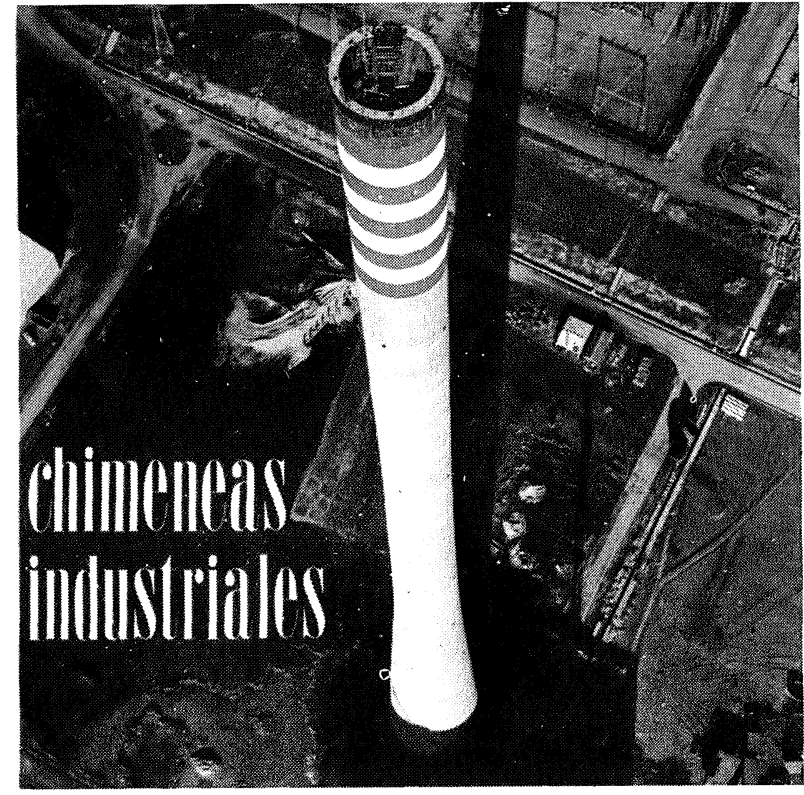

\section{INTRODUCCION}

Las construcciones de gran altura han constituido siempre un reto para la ingeniería civil, de forma que tanto los métodos de cálculo cuanto los constructivos han alcanzado niveles técnicos muy considerables.

Un grupo caracteristico de estas obras es el de aquellas cuya estructura resistente es de sección anular, como son:

Las torres de telecomunicación, que pertenecen a un campo de la Arquitectura profundamente ligado a la Ingeniería.

Las chimeneas industriales, que con la instalación de las grandes centrales térmicas, han requerido alturas realmente importantes.

En esta parcela de la edificación en general, España, gracias a unas pocas empresas especializadas, se encuentra al nivel de los paises más avanzados; avalando esta afirmación las más de una docena de chimeneas que superan los $200 \mathrm{~m}$ de altura construidas en España durante los últimos quince años.

\section{LAS CHIMENEAS INDUSTRIALES}

De estas construcciones altas de sección anular, las chimeneas industriales presentan un cierto número de singularidades en su diseño, cálculo y ejecución como son:

- Importantes solicitaciones térmicas.

- Elevados caudales de gases a evacuar, en ocasiones altamente corrosivos.

- Gran contenido de elementos contaminantes (polvo, $\mathrm{SO}_{2} \ldots$ ) que crean un grave problema de protección del medio ambiente.

La importancia relativa de estos factores singulares ha variado en los últimos años. Las exigencias ecológicas han originado como primera consecuencia una mayor altura media de las chimeneas. Por otro lado la instalación de grandes centrales térmicas, con chimeneas que sirven a dos o más grupos de elevada potencia, hace que los vo- 
lúmenes de gases a evacuar sean enormes, con el consiguiente aumento de los diámetros de los conductos de evacuación de humos que la chimenea aloja en su fuste de hormigón.

Con las tendencias de ahorro energético y recuperación calorífica de los gases evacuados, éstos se lanzan a la atmósfera a temperaturas cada vez más bajas. La primera consecuencia es que en muchas ocasiones se alcanza y se está por debajo del «punto de rocío» de los gases, produciéndose en la propia chimenea condensaciones ácidas que aumentan considerablemente el ataque químico.

Esta disminución de temperatura de los gases infiere también en los problemas ambientales, pues a igualdad de los demás factores que influyen en la dispersión de los gases, cuanto menor es la temperatura de los gases tanto menor es la sobreelevación del penacho de humos y por consiguiente la altura efectiva de la chimenea.

Todo lo anteriormente expuesto hace que cada proyecto de una nueva chimenea adquiera una singularidad que, en el transcurso de este artículo, se intenta describir en forma esquemática.

\section{EL PROYECTO}

En primer lugar se recopilan los datos que constituirán el conjunto básico de datos de partida y que está formado por:

- Datos sobre los gases a evacuar:

- Caudales y temperaturas.

- Composición quimica. Gases contaminantes.

- Viscosidad y calor especifico.

- Datos sobre la zona de ubicación de la chimenea:

- Altitud.

- Temperaturas ambientales.

- Rosa de vientos por velocidades.

- Rosa de vientos por estabilidades.

- Velocidad básica del viento de diseño.

- Coeficiente sísmico básico.

- Estudio geotécnico del terreno.

- Datos sobre los materiales a emplear:

- Resistencias caracteristicas.

- Pesos especificos.

- Conductividades térmicas.

En la mayoria de los casos, los cálculos que se realizan son de comprobación; es decir, suponiendo "a priori» que la construcción tendrá unas caracteristicas determinadas, se calcula el efecto que las solicitaciones produci- rán en ella y se comprueba si dichos efectos cumplen una serie de valores condicionantes - generalmente recogidos en Normas internacionales- que se denomina "conjunto de limitaciones de proyecto".

Los cálculos a realizar son siempre de gran complejidad. Hasta tal punto que en numerosas ocasiones resultan inabordables con métodos manuales. Pero aún en el caso de poder ser realizados normalmente, resulta en la práctica imposible que las caracteristicas supuestas de la construcción se confirmen como las óptimas en la primera comprobación. Ello obliga a modificar los supuestos iniciales y después de un nuevo cálculo, comprobar el cumplimiento de las limitaciones del proyecto. Repitiéndose el proceso tantas veces como sea necesario para conseguir un diseño optimizado.

Todo lo anteriormente expuesto hace imprescindible el uso de ordenadores para conseguir proyectos técnicamente aceptables, comercialmente competitivos y cuya realización se lleve a cabo con plazos cada vez más cortos.

\section{LOS MEDIOS INFORMATICOS}

Existen evolucionados sistemas de cálculo y diseño asistidos por ordenador, que permiten al proyectista construir modelos matemáticos capaces de comportarse de forma muy similar a como lo hará posteriormente la estructura real.

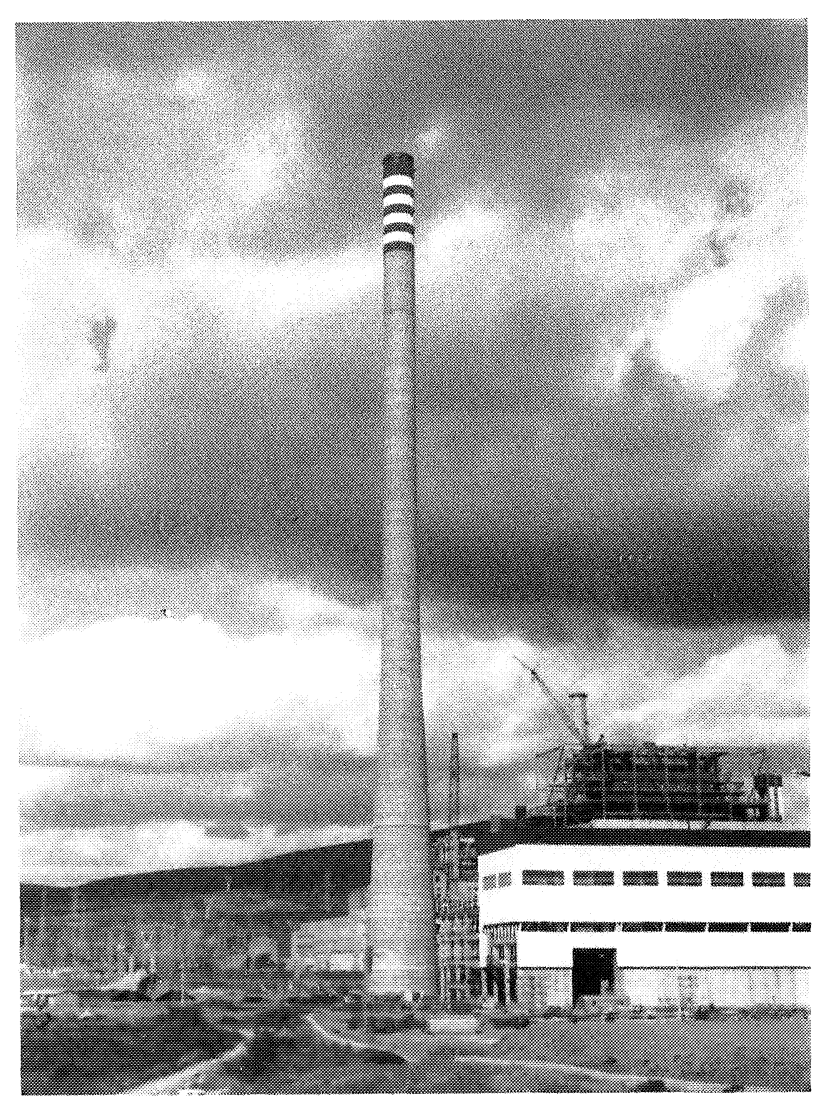


Los sistemas de diseño asistido por ordenador están desarrollados sobre la base de mantener un intenso diálogo proyectista-ordenador, pudiendo el primero dirigir el flujo de cálculos como su experiencia le dicte, seleccionando el orden en que deben ser efectuados, obtener resultados parciales con objeto de verificar la validez del modelo construido, simular situaciones hipotéticas e introducir los datos reales del proyecto.

Después de un trabajo breve y confortable, el proyectista llega a obtener resultados satisfactorios. Es entonces cuando solicita del ordenador la impresión sobre papel de los documentos de cálculo que configurarán la memoria del proyecto.

Para un sistema con estas características es necesario disponer de un terminal interactivo conectado al ordenador. Dicho terminal se compone de una pantalla gráfica o alfanumérica a través de la cual se envian los mensajes en ambas direcciones (proyectista-ordenador y ordenador-proyectista), un lápiz - o pistola - electrónico permite seleccionar con precisión y rapidez el punto de la pantalla de diálogo sobre el que se desea escribir y un teclado similar al de una máquina de escribir mediante el que el proyectista escribe sus mensajes.

$\mathrm{Al}$ otro extremo de la línea de transmisión, el ordenador destina algunos recursos a esta tarea: una cierta zona de su memoria aloja los programas, durante algún tiempo las unidades de control y cálculo se dedican a este trabajo, los soportes magnéticos (discos) contendrán datos y resultados intermedios durante el tiempo que dure el pro-

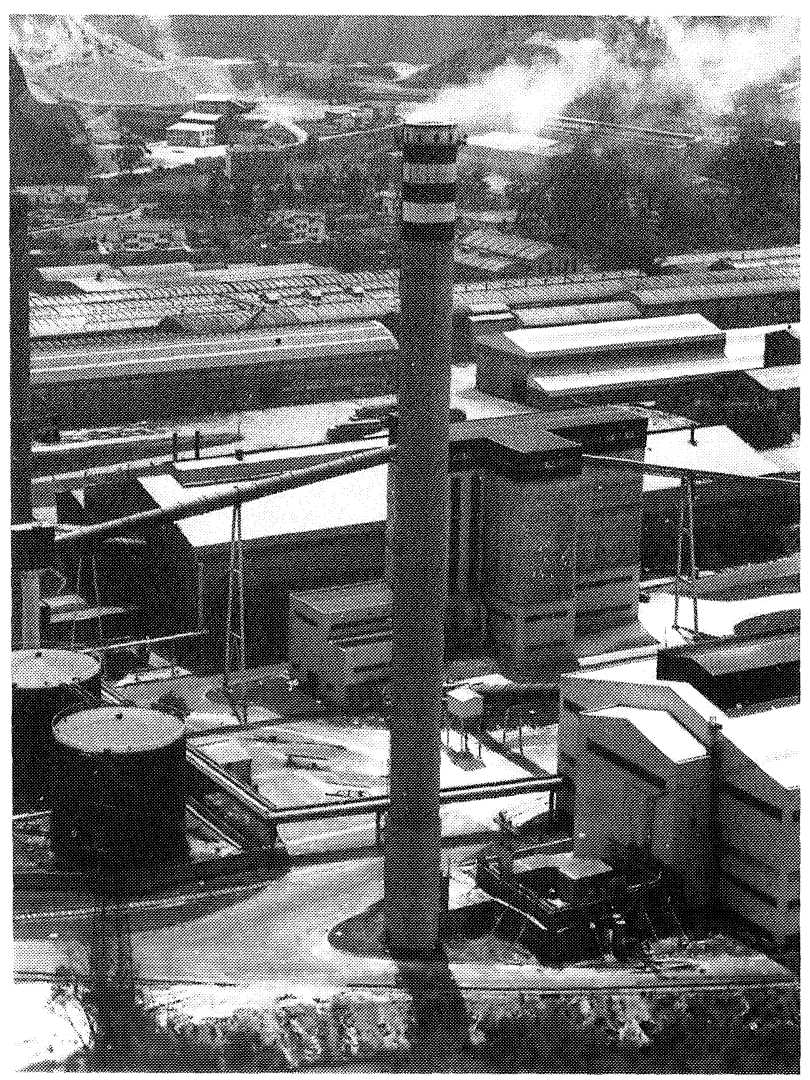

ceso y archivos históricos permanentes, en los que irá quedando almacenada la información que refleja la experiencia y que posteriormente podrá ser utilizada para la realización eficaz de nuevos proyectos. Por último, el ordenador pone a disposición del proyectista una rápida impresora a través de la cual obtendrá los documentos justificativos del cálculo.

El sistema lógico que efectúa el proceso de cálculo se compone de un cierto número de programas que son agrupados en bloques de acuerdo con su finalidad:

1. Cálculo de la altura de la chimenea.

2. Cálculo del diámetro del conducto de evacuación de humos.

3. Composición del conducto de evacuación de humos.

4. Cálculo del diámetro, espesores, armaduras del fuste o pared resistente de hormigón armado.

5. Cálculo de cimientos (dimensiones, armaduras).

6. Aberturas de ventilación de la cámara existente entre el fuste y el conducto de humos.

7. Cálculo de las placas interiores que sirven de soporte al conducto de humos.

8. Comprobaciones especiales:

a) Velocidad critica de oscilación de la estructura.

b) Efectos de seísmo.

\section{EL CALCULO}

En una chimenea industrial se pueden diferenciar cuatro partes fundamentales:

- Conducto de evacuación de humos, que se denomina revestimiento.

- Placas anulares para el apoyo del revestimiento.

- Estructura resistente o fuste.

- Cimentación.

- El revestimiento tiene como misión la protección del hormigón del fuste del ataque químico y térmico de los humos, por ello su composición es a base de capas de materiales antiácidos y aislantes.

El revestimiento, sobre todo en chimeneas de gran altura, se fracciona en tramos de longitudes medias de 20 metros. Estos tramos se solapan, como sucede en las canalizaciones de agua, gas, etc., en forma tal que se consigue una buena estanquidad con la menor alteración del régimen del fluido, y por tanto con una pérdida de carga muy pequeña.

- Las placas anulares de hormigón sirven de apoyo a los distintos tramos del revestimiento. La situada en coronación cubre el espacio entre el fuste y el revestimiento, y la inferior hace también de losa cenicero. 


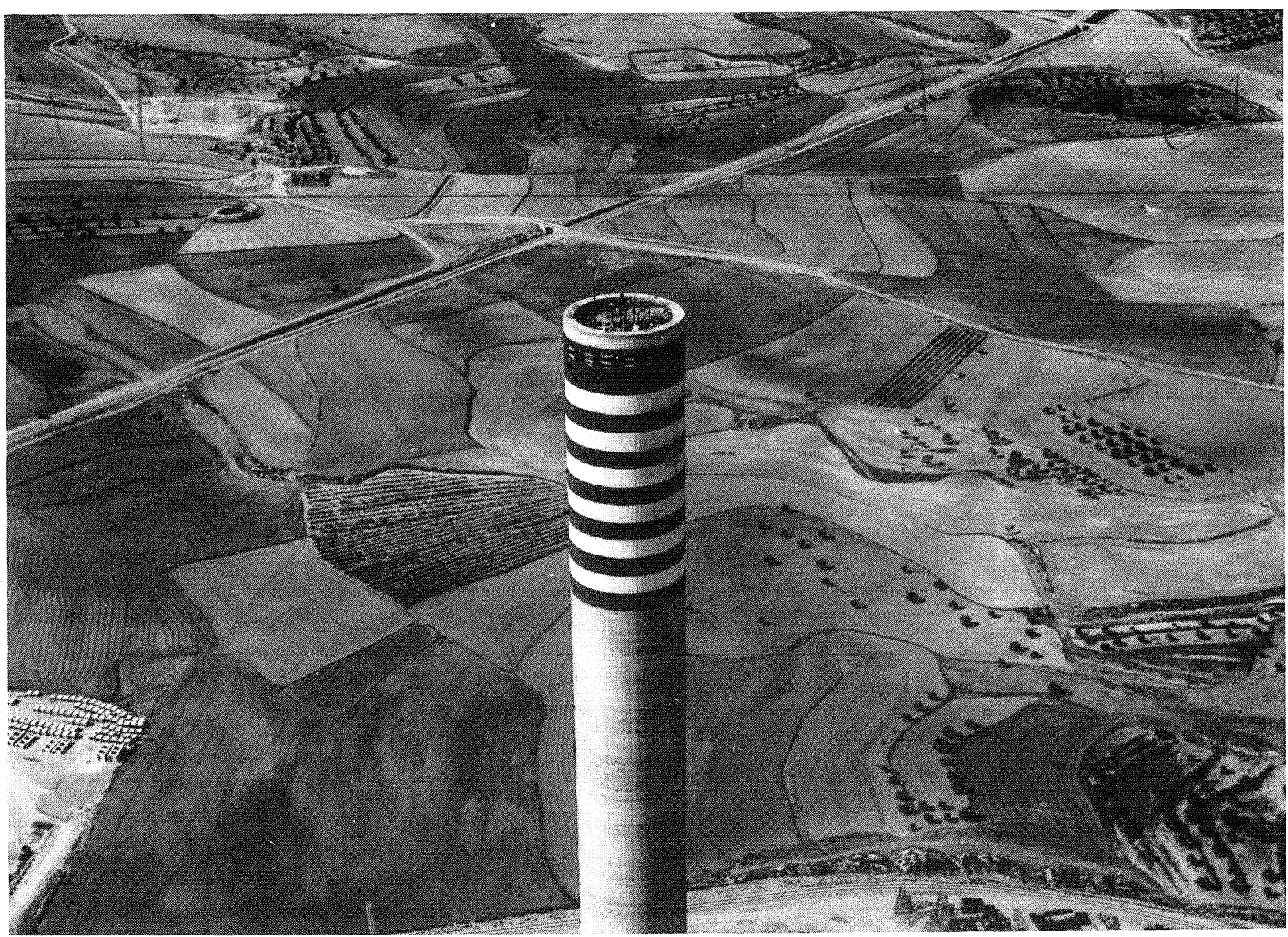

Estas placas se apoyan en ménsulas discontinuas que se disponen convenientemente en el fuste.

Las placas anulares están perforadas por orificios que permiten el paso del aire de ventilación y el acceso mediante escalera interior y ascensor en algunos casos significativos.

Entre el revestimiento y el fuste se dispone de un espacio ventilado y transitable, con un ancho libre mínimo de $80 \mathrm{~cm}$ y que se denomina cámara de ventilación.

Dos son los objetivos básicos que se persiguen con el establecimiento de esta cámara.

Eliminación del riesgo de ataque químico de los condensados sobre el hormigón, gracias al arrastre de las posibles filtraciones de humos por la corriente del aire de ventilación.

Inspección del interior del fuste y del revestimiento, puesto que la cámara es perfectamente transitable y su temperatura es del orden de 30 a $35^{\circ} \mathrm{C}$, cuando la temperatura exterior a la chimenea es del orden de $20{ }^{\circ} \mathrm{C}$.

- El fuste, de hormigón armado, es el encargado de absorber y canalizar hasta la cimentación todos los esfuerzos debidos a su peso propio, peso del revestimiento y placas, viento, sísmico y térmicos.

En el fuste se disponen las aberturas necesarias para entrada y salida del aire de ventilación, entradas de los canales de humos, puertas de acceso y orificios para instalación de balizas de señalización aérea, aparatos para toma de muestras, etc.

- La cimentación, que es la encargada de recoger las acciones que le transmite el fuste y distribuirlas en el terreno.

En los siguientes apartados se describen las características geométricas y funcionales de cada uno de los elementos aqui citados, así como su procedimiento usual de cálculo. 


\section{DIMENSIONES FUNDAMENTALES}

El primer paso a dar en el diseño de una chimenea industrial consiste en fijar las dimensiones fundamentales que por si mismas definen la chimenea:

- Diámetro del conducto de evacuación de humos.

- Altura de la chimenea.

\section{ESQUEMA DEL MODELO DE DIFUSION}

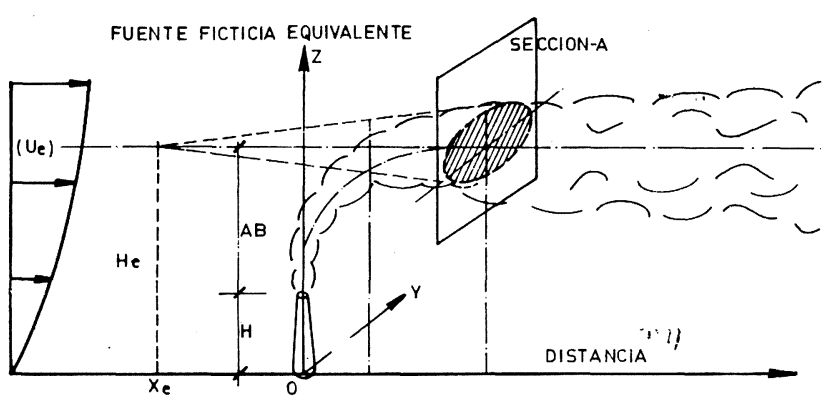

$H=$ ALTURA CHIMENEA Ue = VELOCIDAD DEL VIENTO

$A B=$ ALTURA DEL PENACHO WO VELOCIDAD EVACUACION HUMOS

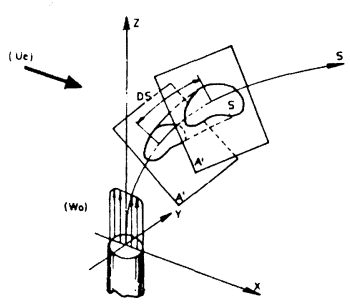

a) Determinación del diámetro libre del conducto de evacuación de humos.

Se disponen como datos de partida de los distintos regímenes de funcionamiento de los hornos y calderas que evacúan sus humos a través de la chimenea.

Los regímenes de funcionamiento más característicos son:

- normal de operación,

- mínimo de operación y

- emergencia o «test block».

Para cada uno de ellos se establece un intervalo correcto de velocidades de evacuación de humos.
Velocidad de evacuación de humos; en metros por segundo:

- $16 \mathrm{~m} / \mathrm{s}$ a $22 \mathrm{~m} / \mathrm{s} \ldots \ldots$. Normal de operación;

- $6 \mathrm{~m} / \mathrm{s}$ a $12 \mathrm{~m} / \mathrm{s} \ldots$.... Mínimo de operación;

- $28 \mathrm{~m} / \mathrm{s}$ a $34 \mathrm{~m} / \mathrm{s}$............ Emergencia.

Estos límites están fijados empíricamente considerando que para velocidades superiores a los $34 \mathrm{~m} / \mathrm{s}$ pueden presentarse problemas acústicos $\mathrm{y}$ de erosión.

La velocidad entre 16 y 22 metros por segundo es la que se considera óptima.

Por último el límite inferior de velocidades se establece para que no aparezcan problemas de reflujo a la salida de los humos con el consiguiente incremento del ataque químico sobre el hormigón de coronación de la chimenea.

De este modo se obtienen unos diámetros libres del conducto que encajan dentro de los límites de velocidades citados.

De todos ellos, se toma como diámetro libre definitivo el menor de ellos que cumpla con las exigencias de tiro que cada horno o caldera imponga.

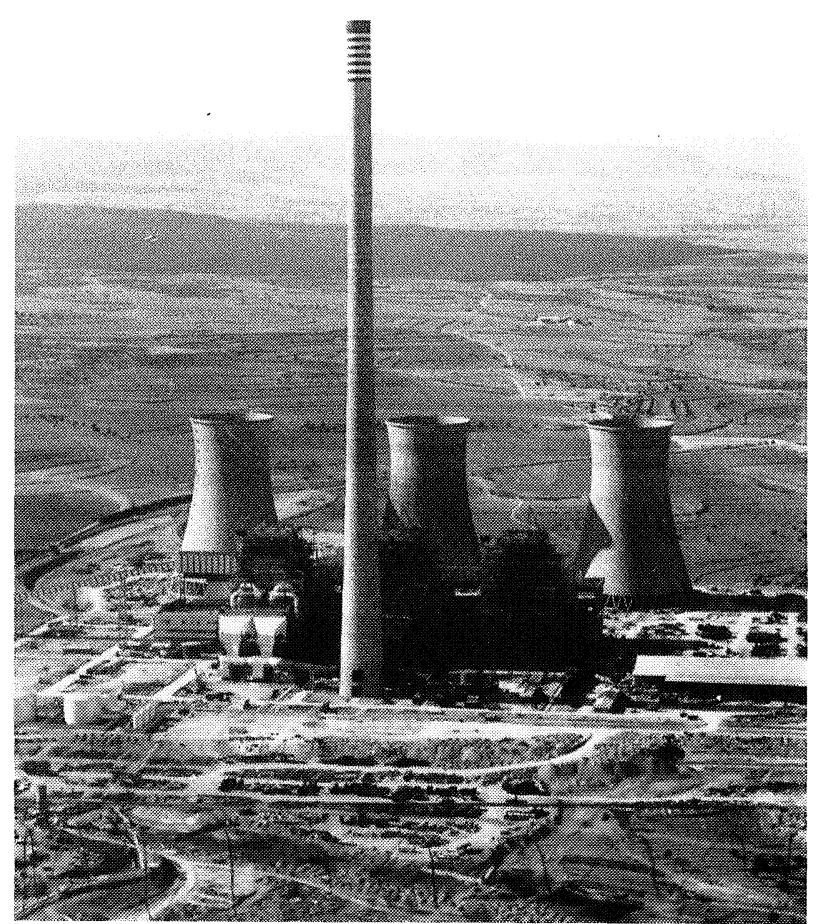


Este cálculo es de carácter iterativo y exige muchas pasadas por el ordenador, estableciendo los tiros netos en cada horno o caldera que alimente de humos a la chimenea. Es gracias al medio de cálculo electrónico, que este proceso puede ser realizado en un plazo relativamente breve, determinando el diámetro óptimo del conducto de evacuación de humos.

b) Determinación de la altura de la chimenea.

En este caso, el diámetro del conducto de evacuación de humos entra a formar parte de los datos de partida para establecer la altura mínima que debe tener la chimenea.

Otros datos previos son el caudal y la temperatura de los humos en su régimen normal de operación.

Al conocer la composición de los humos, sabemos qué tipos y cantidades de contaminantes emite la chimenea, es decir, conocemos los niveles de emisión. Estos niveles en los cálculos de contaminación ambiental suelen expresarse en el caso de las chimeneas industriales en miligramos de $\mathrm{SO}_{2}$ contenidos en cada kilogramo de humos evacuados.

Estos contaminantes se difunden en la atmósfera y pueden medirse a diferentes distancias de la chime-

Relación entre el nivel de inmisión (C) y la distancia de la fuente al punto observado $(X)$, para distintas alturas efectivas $(\mathrm{He})$.

(C) $\left(\mu G / m^{3}\right)$

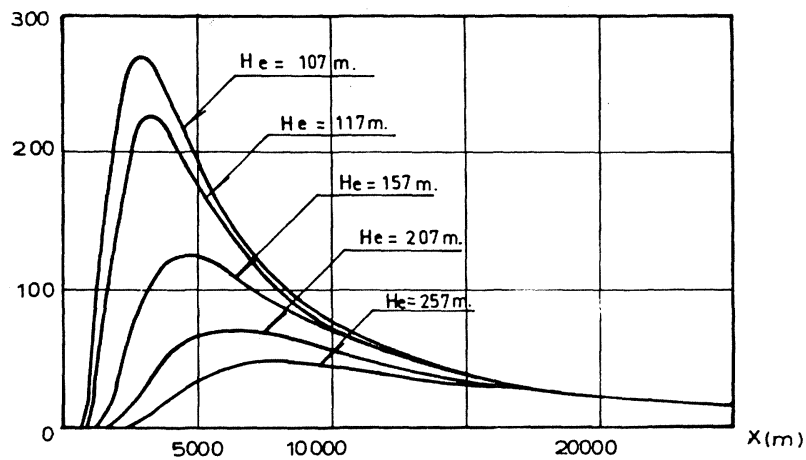

Relación entre el nivel de inmisión (C) y la distancia de la fuente al punto observado (X), para distintas clases de estabilidad atmosférica.

(c) $\omega s / \mathrm{m}^{3}$ )

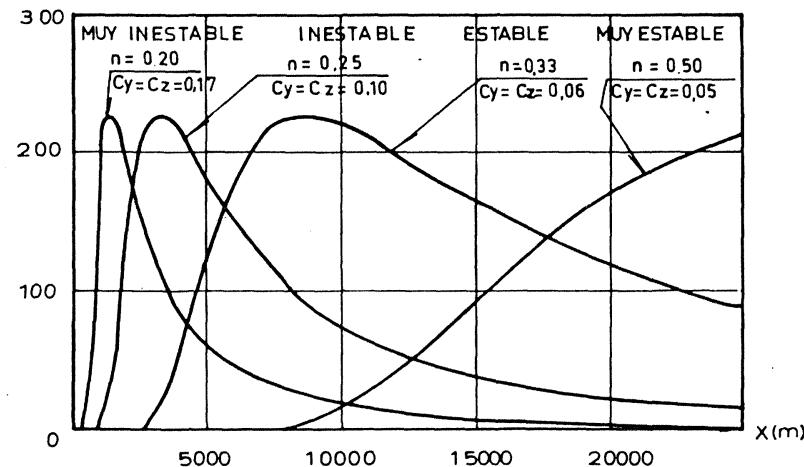

nea unas concentraciones de $\mathrm{SO}_{2}$ que constituyen los niveles de inmisión.

Se comprende fácilmente que las concentraciones de $\mathrm{SO}_{2}$ medidas en cada punto, no son exclusivamente procedentes de la fuente de emisión en estudio, es decir de la chimenea, sino que también proceden de otras fuentes de emisión de zonas próximas.

Por ello el procedimiento que podriamos considerar como normal, para el caso de la instalación de una chimenea sería el siguiente:

- Establecimiento en los puntos que se determinen previamente del entorno de la chimenea de los niveles de inmisión existentes, lo cual se logra mediante las correspondientes mediciones in situ.

- En función de los valores anteriores establecer qué incrementos de inmisión pueden admitirse en cada punto debido exclusivamente a la chimenea, cuya altura pretendemos instalar. Estos valores constituyen el condicionante fundamental que nos permitirá determinar la altura mínima de la chimenea.

Ahora sólo falta establecer la relación existente entre el nivel de emisión y el nivel de inmisión, es decir, un modelo de difusión.

Los niveles de concentración de contaminantes, no sólo vienen determinados por las emisiones sino también por las condiciones locales del terreno (topografia) y la capacidad difusora de la atmósfera.

La ecuación general de difusión de los contaminantes en la atmósfera es:

$$
\begin{aligned}
& x(\mathrm{x}, \mathrm{y}, \mathrm{z}, \mathrm{h})=\frac{\mathrm{Q}}{2 \pi \sigma_{\mathrm{y}} \sigma_{\mathrm{z}} \mathrm{U}}\left[\exp -\frac{1}{2}\left(\frac{\mathrm{y}}{\sigma_{\mathrm{y}}}\right)^{2}\right] \\
& {\left[\exp -\frac{1}{2}\left(\frac{\mathrm{z}-\mathrm{h}}{\sigma_{\mathrm{z}}}\right)^{2}\right]+\left[\exp -\frac{1}{2}\left(\frac{\mathrm{z}+\mathrm{h}}{\sigma_{\mathrm{z}}}\right)^{2}\right]}
\end{aligned}
$$

En la anterior fórmula intervienen los siguientes conceptos:

$$
\begin{aligned}
X(\mathrm{x}, \mathrm{y}, \mathrm{z}, \mathrm{h})= & \text { Concentración de contaminantes (nivel } \\
& \begin{array}{l}
\text { de inmisión) en el punto definido por } \\
\text { sus coordenadas } \mathrm{x}, \mathrm{y}, \mathrm{z}, \text { para una altura } \\
\text { efectiva de emisión } \mathrm{h} .
\end{array} \\
\mathrm{=} & \text { Nivel de emisión de contaminantes. } \\
\mathrm{Q} & \text { Velocidad del viento. } \\
\mathrm{U} \quad & \text { Altura efectiva de emisión de la chime- } \\
& \text { nea, es decir, altura geométrica más al- } \\
& \text { tura por sobreelevación del penacho } \\
& \text { de humos. }
\end{aligned}
$$


ROSA DE VIENTOS: Distribución por frecuencia de direcciones y velocidades del viento.

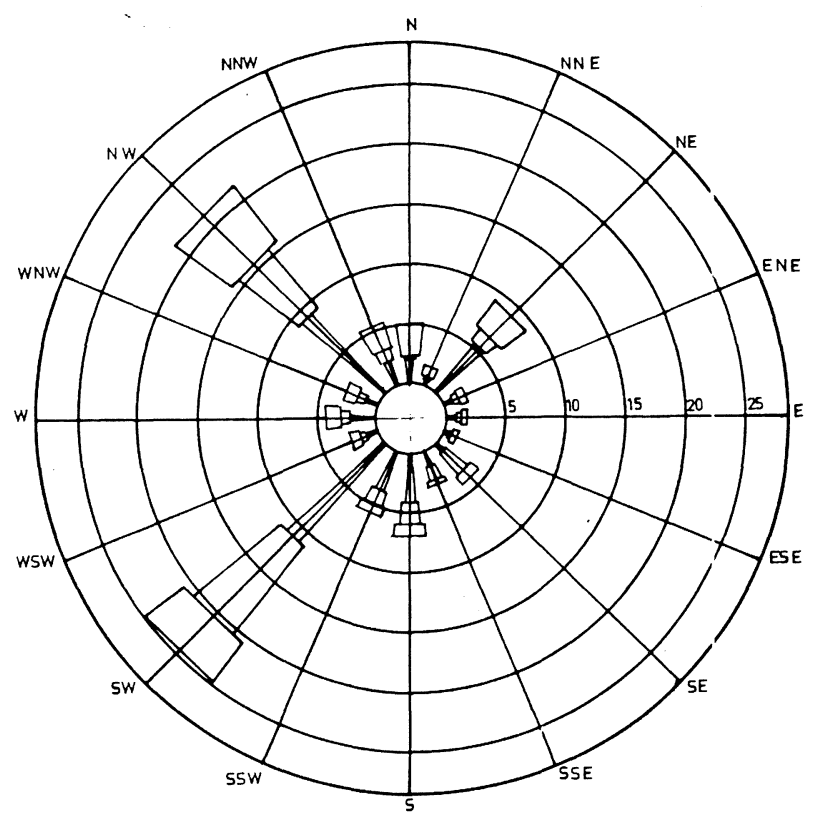

SITUACIONES

INESTABLES A-B-C

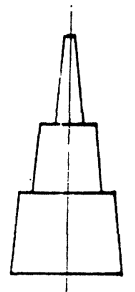

ESTABLES E-F

$\sigma_{\mathrm{y}}, \sigma_{\mathrm{z}} \quad=$ Parámetros de dispersión, que son función de las caracteristicas térmicas y dinámicas de la atmósfera.

Queda por definir, para poder completar el proceso de cálculo, las características térmicas y dinámicas de la atmósfera. Esto se consigue mediante el establecimiento de la Rosa de Vientos, y las clases de estabilidad atmosférica.

La Rosa de Vientos es una distribución por frecuencias de direcciones y velocidades del viento.

Para las clases de estabilidad atmosférica, cuando se dispone de datos metereológicos recogidos en estaciones metereológicas, se suele adoptar el criterio de Pasquill.

En la tabla se recogen las distintas clases de estabilidad adoptadas.

Con los datos recogidos en la estación metereológica, se componen los cuadros de frecuencias relativas expresadas en tanto por ciento de simultaneidad de dirección y velocidad del viento, junto a la clase de estabilidad atmosférica. Estos cuadros se confeccionan para períodos mensuales y anuales.

El procedimiento de cálculo es iterativo, se supone una altura de chimenea, se adopta una formula de so-
Clasificación de la atmósfera según su estabilidad conforme al criterio establecido por Pasquill

\begin{tabular}{|c|c|c|c|c|c|}
\hline \multirow[t]{2}{*}{$\begin{array}{l}\text { Velocidad del viento } \\
\text { a } 10 \mathrm{~m}(\mathrm{~m} / \mathrm{s})\end{array}$} & \multicolumn{3}{|c|}{$\begin{array}{c}\text { Día } \\
\text { (Radiación solar incidente) }\end{array}$} & \multicolumn{2}{|c|}{$\begin{array}{c}\text { Noche } \\
\text { (Cubierta de nubes) }\end{array}$} \\
\hline & Fuerte & Moderada & Débil & $>4 / 8$ & $<\mathbf{3 / 8}$ \\
\hline$<2$ & A & A-B & B & - & - \\
\hline $2 \cdot 3$ & A-B & B & $\mathrm{C}$ & $E$ & $\mathrm{~F}$ \\
\hline $3 \cdot 5$ & B & B-C & $\mathrm{C}$ & D & $\mathrm{E}$ \\
\hline $5 \cdot 6$ & $\mathrm{C}$ & C-D & D & D & D \\
\hline$>6$ & $\mathrm{C}$ & D & D & D & D \\
\hline
\end{tabular}

La radiación solar es fuerte cuando la altura del Sol es $>60^{\circ}$, es moderada si está comprendida entre 35 y $60^{\circ}$ y es débil si es inferior a $35^{\circ}$.

breelevación del penacho de humos, y se obtiene la altura efectiva de emisión.

Con los datos metereológicos y fórmula de difusión ya descrita se realiza un primer cálculo, obteniéndose como resultados las concentraciones relativas medias diarias de $\mathrm{SO}_{2}$ en $\mu \mathrm{g} / \mathrm{m}^{3} \mathrm{~N}$, así como los valores medios mensuales y anuales.

Finalmente se dispone de los criterios legales de calidad del aire.

El cálculo se repite, modificando la altura de la chimenea hasta conseguir los niveles de contaminación admisibles.

El procedimiento es laborioso y extremadamente largo, siendo prácticamente imposible su realización sin medios de cálculo electrónico.

\section{DIMENSIONAMIENTO ECONOMICAMENTE OPTIMO DE LA CHIMENEA}

Establecidas las dimensiones fundamentales de la chimenea, el cálculo se encamina a la determinación de las características cualitativas y dimensionales de los materiales que constituirán el revestimiento, fuste, placas y cimentación de la chimenea.

\section{- Revestimiento}

Conocidas las características de los humos se determinan los materiales óptimos para hacer frente al ataque químico y térmico de los mismos. El primero condiciona el carácter antiácido del material y el segundo su poder aislante.

La misión fundamental del revestimiento es proteger al fuste de hormigón, que es la estructura resistente, y conseguir en la cámara intermedia de ventilación una temperatura tal que permita sea transitable.

Cuando la temperatura de los humos es alta, por encima de los $250^{\circ} \mathrm{C}$, el gradiente térmico que debe sufrir el revestimiento puede ser grande, sobre todo en los arranques y puesta en marcha, por lo que interesa ir a 
un revestimiento multicapa, que con capas de relativamente débil espesor, 7-8 cm, permita absorber dicho gradiente con tensiones térmicas no elevadas.

En los cálculos térmicos, realizados con auxilio del ordenador, juegan papel importante: la curvatura de la pared del conducto, las temperaturas límites de empleo de sus materiales, los coeficientes de conductividad y transferencia térmica, etc.

\section{- Fuste}

Para el dimensionamiento del fuste se parte de los siguientes datos:

- Altura de la chimenea.

- Diámetro y espesor del conducto de revestimiento.

- Ancho mínimo de la cámara ventilada.

- Norma de dimensionamiento: ACI, DIN-1056, CICIND.

- Caracteristicas de los materiales: Resistencia característica, límite elástico, coeficiente de dilatación, módulo de elasticidad.

- Teoría de dimensionado: En tensiones admisibles, en rotura.

- Precios de hormigón, acero y encofrado, necesarios para el dimensionamiento económicamente óptimo.

- Acciones a considerar: Peso propio, viento, terremoto, soleamiento, gradiente térmico, momentos de segundo orden.

- Definición de las secciones en que se desea la obtención de esfuerzos, generalmente cada $20 \mathrm{~m}$ y todas las que tienen singularidades como placas de hormigón, aberturas, etc.

- Definición de las aberturas de la chimenea.

Como parámetros de cálculo se consideran los valores mínimos de armaduras, tanto horizontal como vertical, las constantes para el cálculo de los esfuerzos de ovalización, los espesores mínimos y máximos que deben considerarse para la pared del fuste según la norma adoptada, y las limitaciones en las tensiones o deformaciones que, para cada tipo de acción, se establecen conforme a la teoría de dimensionado y a la norma aplicada.

El cálculo electrónico establece automáticamente un estudio comparativo de tipos para distintas geometrías del fuste (fuste cilíndrico, fuste parabólico, fuste cilíndrico y parabólico) con distintas leyes de variación del diámetro exterior del fuste.

Para cada uno de ellos realiza el cálculo optimizado definiendo en resumen: $\mathrm{m}^{3}$ de hormigón, $\mathrm{m}^{2}$ de encofrado y $\mathrm{t}$ de acero.
Se establece así el tipo óptimo de chimenea, para el cual y en cada sección considerada se obtienen los siguientes resultados:

Diámetro exterior, espesor de pared, cuantias vertical y horizontal de armaduras, en forma de $\varnothing$ de barra y separación entre ellas, así como el número de barras verticales a colocar entre cada dos pórticos de encofrado. Tensión en el hormigón y la armadura. Y finalmente, la valoración económica de la chimenea, desglosada en: fuste, placas y revestimiento.

Con el dimensionamiento de la cimentación e incorporación del coste de la misma, se dispone de la valoración total de la chimenea.

\section{- Cimentación}

Conocidas ya las geometrias del fuste y conducto, asi como las acciones transmitidas a la cimentación, se procede al dimensionamiento y valoración de la misma.

Este cálculo no encierra ninguna singularidad digna de mención. Tan sólo destacaremos como importantes la obtención del máximo asiento diferencial entre dos puntos extremos de un diámetro. Este valor se introduce como una acción más en el cálculo del fuste de la chimenea.

\section{- Cálculo de las aberturas de ventilación}

El conducto de revestimiento se fracciona en tramos que se apoyan en unas placas anulares de hormigón.

Estas placas son un obstáculo para el paso del aire en la cámara intermedia de ventilación, por ello es necesario disponer en las mismas unos orificios que permitan la circulación por tiro natural del aire de ventilación de forma que la temperatura en la cámara esté en los valores admisibles prefijados.

En forma simplificada el concepto es el siguiente:

Los gases calientes ascienden por el interior del revestimiento, el aire de ventilación asciende por tiro natural por la cámara intermedia gracias al calor cedido por los gases a través del revestimiento.

A efectos de análisis se subdivide la chimenea en muchas secciones en sentido vertical estableciéndose en cada una de ellas un equilibrio térmico, con una velocidad del aire de ventilación supuesta.

Este análisis proporciona las temperaturas que en las diferentes secciones tienen las capas del revestimiento y el aire de la cámara de ventilación.

El cálculo comienza en la base de la chimenea donde se conocen las temperaturas de los gases y del aire de ventilación a su entrada en la chimenea. Dicho cálcu- 
lo se repite sección tras sección. Una vez conocida la distribución de temperaturas del aire de ventilación podemos conocer su velocidad real y compararla con el valor supuesto, si ambos valores son distintos se repite el cálculo en forma iterativa hasta conseguir una aproximación satisfactoria.

En las placas de hormigón se determinan el número y dimensiones de orificios necesarios para el paso del aire, a la vez que se computan las pérdidas de carga que se producen al paso del aire por dichos orificios.

Por último se establecen el número de orificios necesarios para entrada del aire de ventilación, en la base de la chimenea, así como las de expulsión del aire caliente en coronación de la chimenea.

La limitación que se introduce en este cálculo es que la temperatura del aire de ventilación en la cámara intermedia no sobrepase, en ninguna sección de la misma, la considerada como admisible.

\section{- Cálculo de las placas interiores de soporte del conducto de revestimiento}

Este cálculo no se realiza mediante diálogo con pantalla sino por el procedimiento tradicional de tarjetas perforadas, usual en el cálculo de un emparrillado plano.

\section{- Comprobaciones especiales}

Las comprobaciones especiales que usualmente se realizan en el cálculo de una chimenea son las siguientes:

- Determinación de las velocidades críticas de oscilación de la estructura. Vibraciones transversales a la dirección del viento (Torbellinos de Von Karman).

- Determinación de esfuerzos en una sección circular sometida a una distribución horizontal de cargas de viento cualquiera. Así obtendremos los parámetros necesarios para el cálculo de la ovalización.

- Simulación sísmica.

- Comprobación de tensiones en las capas del conducto en régimen no estacionario de temperaturas.

\section{CONCLUSION}

Con la anterior descripción de las distintas fases de un diseño de una chimenea industrial de las que hoy día se construyen para las grandes centrales térmicas y para las modernas refinerias, se trata de llamar la atención sobre la importancia del proyecto y la necesidad de que éste sea llevado a cabo por ingenieros especialistas en este campo de la edificación industrial.

\section{Bibliografia}

GÖTZEN, W.: «Schornsteine in Massivbauweise». VulkanVerlag. Essen, 1976.

BOTTENBRUCH y KÄMMER: «Immissionsabsenkung durch optimal dimensionierte schornsteine». Technisch-Wissenschaftliche Informationen. NR. 7, julio, 1978.

BOTTENBRUCH y BÖHMER: «Moderner Schornsteinbau». Beton-Informationen NR. 5, Düsseldorf, 1977.

PARRA y DEL SOLAR: "Obras de gran altura». Obras. Revista de Construcción, n. ${ }^{\circ}$ 141. Madrid, 1981.

$$
\text { is is }
$$

